\title{
Energy Efficient Topology Control Protocol for Wireless Sensor Networks
}

\author{
Hagar Noori ${ }^{1}$, Arash Nikdel ${ }^{2}$ and Mahdi Mosleh ${ }^{3}$ \\ ${ }^{1,2,3}$ Department of Computer Engineering, Andimeshk Branch, Islamic Azad \\ University, Andimeshk, Iran \\ hagar_noori@yahoo.com ${ }^{1}$,nikdelarash@yahoo.com ${ }^{2}$,mahdi_mosleh@yahoo.com ${ }^{3}$
}

\begin{abstract}
One major problem in the area of Wireless Sensor Networks (WSNs) is the topology control. Topology control protocols in WSNs construct an optimized network topology structure to satisfy the application requirements, such as network connectivity and coverage. In this paper, we deal with the topology control problem by adjusting the ransition radius of the sensor nodes. We produce a cluster-based topology control scheme and propose a new Energy Efficient Topology Control Protocol called EETCP. In this protocol, proper transition radius can be determined using Harmony Search (HS) algorithm. The proposed protocol dynamically adjusts transition radius of nodes nlike some previous protocols which should select radius values among predefined values.). Thus, the proposed protocol has some advantage compared to the previous protocols. EETCP has less average number of neighbors compared to the existing protocols. Also, the energy, consumption in EETCP is less than other protocols and the network lifetime $12 \mathrm{e}$ be prolonged. In addition, the network connectivity in EETCP is in the acceptable level. The probosed protocol is simulated and the above advantages are demonstrated by the simulation results.
\end{abstract}

Keywords: Energy Consumption, Harmony Search Algorithm, Topology Control, Wireless Sensor Network

\section{Introduction}

The Wireless Sensor Networks (WSNs) have a wide range of applications, for instance, habitat monitoring, battlefield surveillance, control and automation, environmental monitoring, healtheare, Security and surveillance [1,3]. The nodes in WSNs are usually powered by battefies, with finite capacity and it is always impossible to replenish the power [10]. Therefore, the applications are hindered by limited energy supply, and one design challenge in sensor networks is to save limited energy resources to prolong the network lifetime [10.17, 24]. Power saving techniques can generally be classified in two categories [10]:

- Scheduling the sensor nodes to alternate between active and sleep mode

- Adjusting the transmission or sensing radius of the wireless nodes

Different applications have different requirements, so they adopt the different network models and design assumptions, such as: network structure, sensor deployment strategy, sensing model, and transmission range [12]. Furthermore, applications differ in their requirements; therefore the sensor networks usually have different design objectives. In [12], 
these design objectives summarized such as: maximizing network lifetime, sensing coverage, and network connectivity.

One major problem in the area of WSNs is the topology control. Topology control protocols in WSNs construct an optimized network topology structure to satisfy the application requirements, such as network connectivity and coverage [12]. Choosing appropriate topology for a sensor network has much effect on networks performance, especially considering power consumption and lifetime network [19]. In this paper, we deal with the topology control problem by adjusting the transition radius of the sensor nodes. We produce a cluster-based topology control scheme, and propose a new Energy Efficient Topology Control Protocol called EETCP. In this protocol, proper transition radius can be determined using Harmony Search (HS) algorithm.

The remaining of the paper is organized as follows: In section 2 we present the related work in the context of topology control in WSNs. In Section 3 we illustrate the harpony search algorithm briefly. Section 4 presents the model and the assumptions made and Section 5 describes the proposed algorithm. Section 6 presents some simulation results and evaluates the algorithm for different sensor field deployments. The paper concludes with Section 7.

\section{Related Works}

So far, many protocols have been introduced for topology control in WSNs. Topology control protocols are divided into homogeneous and heterogeneous topology control protocols [19]. In homogeneous topology control, all network nodes use the same transition radius and topology control problem is to find a minimum value for transition radius considering the network characteristic such as network comectivity and coverage [19]. Homogeneous mode (HOM) [20] and COMPOW [15] are two examples of these methods. The basic idea in $C O M P O W$ is to apply a uniform trans mission power to all sensor nodes, and minimize the power without decreasing network connectivity D2]. When the sensor nodes are uniformly deployed, COMPOW can have a good performance. But, when the sensor nodes are not uniformly deployed, the effieiency of COMPOW decreases, because some nodes will lead all nodes to use large transtrission range, which results in more energy consumption [12].

In heterogeneous topology control, the sensor nodes can have non uniform transition radius [19]. In this group, protocols with information used for making topology are divided into three subgroups. The first suggroup consists of methods based on location. In this subgroup, nodes are informed of their location. Rodoplu and Ming $(R \& M)$ [18] and Local Minimum Spanning Tree (LMST) NH] are two examples of these methods. The second subgroup consists of methods based on orientation. In these methods, nodes don't have exact information of their location, but they can identify direction of their neighbors. Protocol Cone Based Topology Control (CBTC) [22] is an example of these methods. Third subgroup is methods based on neighbors. In these methods, nodes have limited information about their neighbors This information consists of ID number, and distance or quality of node's neighbors Extreme Topology Control (XTC) [23] and $k$-neighbors (Kneigh) [4] are examples in this group.

Radius Adaptation Algorithm-2 Level $(R A A-2 L)$ is another topology control protocol. In this protocol, each node chooses one of two transition radius $R_{S}$ or $R_{W}\left(R_{W}<R_{S}\right)$ [21]. If a node with transition radius of $R_{W}$ could communicate with a neighbor with transition radius $R_{S}$, it chooses the transition radius $R_{W}$, else it chooses the transition radius $R_{S}$. In Radius Adaptation Algorithm-3 Level ( $R A A-3 L)$, each node chooses one of three transition radius: $R t, R_{S}$ or $R_{W}$ $\left(R_{W}<R t<R_{S}\right)$. In our previous work [7], we proposed a Genetic Algorithm-based Topology Control protocol for wireless sensor networks called GATC. Like the GATC, the proposed protocol in this paper is based on $R A A-3 L$, but has less overhead. 


\section{Harmony Search Algorithm}

HS algorithm, originated by Geem et al., [6], is based on natural musical performance processes that occur when a musician searches for a better state of harmony [2, 16, 14]. HS has several advantages with respect to traditional optimization techniques such as the following [2]:

- HS imposes fewer mathematical requirements.

- HS is free from divergence.

- HS does not require initial value settings of the decision variables, thus it may escape the local optima.

- HS can handle both discrete and continuous variables.

- The HS algorithm optimization procedure consists of the following five steps [2] [16] [14]:

Step 1: Problem and algorithm parameter initialization Step 2: Harmony memory initialization and evaluation Step 3: New Harmony improvisation Step 4: Harmony memory update Step 5: Termination criterion check

We give the pseudo-code for HS algorithm as follows [14]:

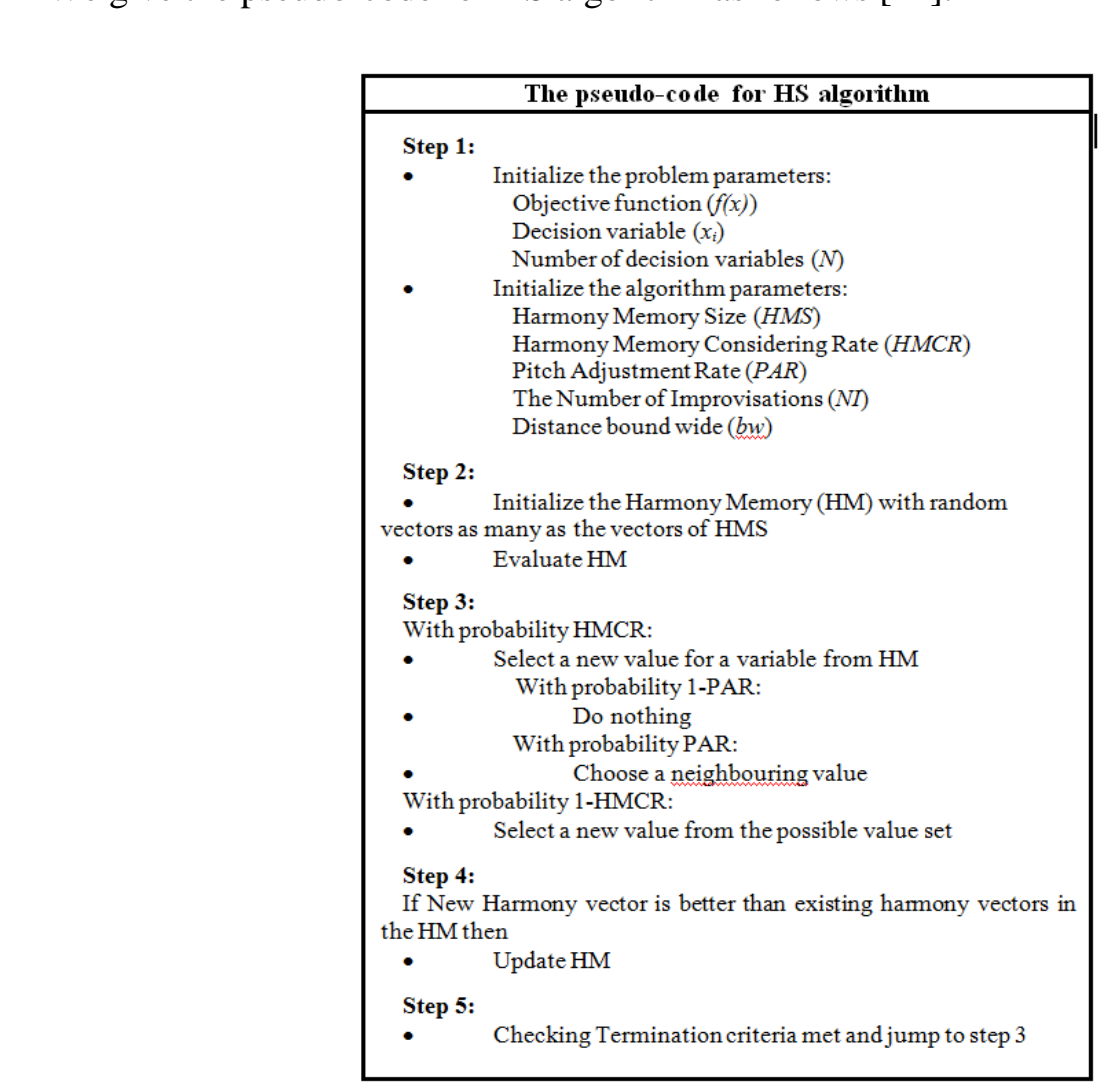

\section{The Network Model and the Assumptions}

In this section, we present the network model and the assumptions used in this paper. 


\subsection{Adjustable Transition Radius}

Define that the sensor set is $S=\left\{n_{1}, n_{2}, n_{3}, \ldots, n_{n}\right\}$ and the transition radius set is $R_{\mathrm{T}}=\left\{R_{1}, R_{2}, R_{3}, \ldots, R_{n}\right\}$, where $R_{i}$ is the transition radius of node $n_{i}$.

We assume that each sensor node ni has adjustable transition radius which can be between a minimum and a maximum transition radius:

- $R_{\text {min }}$ : transition radius with minimum power

- $R_{\text {max }}$ : transition radius with maximum power

- $R_{i}$ : selective transition radius of node $n_{i}$

The value of $R_{i}$ should be between the $R_{\min }$ and $R_{\max }\left(R_{\min } \leq R_{i} \leq R_{\max }\right)$. Value of the transition radius $R_{\min }$ and $R_{\max }$ will be calculated based on $R t$. Value of transition radius $R t$ is determined proportional to the network density [20].

When the distance of both nodes is less than $R_{\max }$, we assume they are neighbor. Each node has three different neighbor sets. Sets of $A_{\min }, A_{T}$ and $A_{\max }$ are obtained by (1):

$$
\begin{aligned}
& \forall n_{i}, n_{j} \in S=\left\{n_{1}, n_{2}, n_{3}, \ldots, n_{n}\right\}, i \neq j: \\
& \begin{cases}n_{j} \in A_{\text {min }}\left(n_{i}\right) & \text { if } \operatorname{Dis}\left(n_{i}, n_{j}\right) \leq R_{\text {min }} \\
n_{j} \in A_{T}\left(n_{i}\right) & \text { if } R_{\text {min }}<\operatorname{Dis}\left(n_{i}, n_{j}\right) \leq R_{i} \\
n_{j} \in A_{\max }\left(n_{i}\right) & \text { if } R_{i}<\operatorname{Dis}\left(n_{i}, n_{j}\right) \leq R_{\text {max }}\end{cases}
\end{aligned}
$$

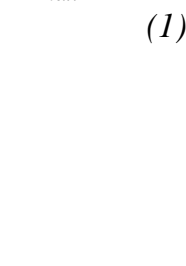

In (1), $A x\left(n_{y}\right)$ shows the $A x$ set of node $n_{y}$ and $\operatorname{Dis}\left(n_{j}, n_{i}\right)$ is the distance between node $n_{i}$ and $n_{j}$. Therefore, for each node $n_{i} \in S=\left\{n_{i} n_{2} n_{3}, \ldots, n\right\}$

$$
\begin{aligned}
& A_{\text {max }}\left(n_{i}\right) \cup A_{T}\left(n_{i}\right) \cup A_{\text {min }}\left(n_{i}\right)=\text { All neighbors of node } n_{i} \\
& A_{\text {max }}\left(n_{i}\right) \cap A_{T}\left(n_{i}\right) \cap A_{\text {min }}\left(n_{i}\right)=
\end{aligned}
$$

We define the number of links for the sensor network:

$$
\text { Number of links }-\sum_{i=1 \text { to } n} \text { All neighbors of node } n_{i}
$$

$$
=\sum_{i=\text { tow }_{1}}\left|A_{\min }\left(n_{i}\right) \cup A_{Y}\left(n_{i}\right) \cup A_{\max }\left(n_{i}\right)\right|
$$

Where $n$ is the number of the sensor. Thus, the average number of neighbors is defined as:

$$
\text { Average numberofneighbors }=\text { Number of Links } / n
$$

Also, the average of transition radius is calculated as:

$$
\text { Average of transition radius }=\sum_{i=1 \text { to } n} R_{i} / n
$$

The main problem in this study is choosing minimum transition radius $R_{i}$ between $R_{\text {min }}$ and $R_{r a x}$ for each node $n_{i} \in S=\left\{n_{1}, n_{2}, \ldots, n_{n}\right\}$ without decreasing the network connectivity.

\subsection{The cluster-based Architecture}

Similar to the cluster-based coverage control scheme introduced in [10], we use a clusterbased topology control scheme in this paper, which is scheduled into rounds. In each round, firstly, the target area is divided into several equal squares. Then the node in each square having the largest energy will be chosen as the cluster-head. The procedure of selecting the cluster-head is the same works in [10] [9]. The cluster-head has full control of the square and 
it will choose transition radius of nodes. In the next round, another sensor set will be selected as the cluster head. So the energy consumption among all the sensors can be balanced well [10].

\subsection{Energy Consumption Analysis}

According to different energy consumption models, the energy consumed by a sensor node to deal with a transmission task is proportional to $r^{2}$ or $r^{4}$, where $\mathrm{r}$ is the transition radius of node $[10,13]$. In this paper, we take the energy consumption of the transition task as $u . r^{2}$, where $u$ is the factor.

$$
E(r)=u \cdot r^{2}
$$

Thus, the energy consumption of the sensor set, which is related to the sum of the sensor's transition radius squared, is defined as [10]:

$$
E_{\text {total }}=E\left(R_{T}\right)=\sum_{i=1 \text { to } n} E\left(R_{i}\right)=u \cdot \sum_{i=1 \text { ton }} R_{i}^{2}
$$

So, the energy consumption per area is shown as the following:

$$
E_{\text {per-Area }}=E_{\text {total }} / A_{\text {area }}=u . \sum_{i=1 \text { ton }} R_{i}^{2} / A_{\text {area }}
$$

Also, for the brief of the energy consumption analysis, we only consider the energy consumed by the transmission function, and do not include the energy consumption of calculations and sensing [10].

\subsection{The Complete Connectivity of the Sensor Network}

In this paper, we will deal with the nodes deployed randomly. Assume that each one knows its own location which can be achieved by using some location system [10, 5].

A WSN can be modeled as a graph $G=(V, E)$, where $V$ is the set of sensor nodes and $E$ is the set of wireless links [8]. The complete connectivity of the sensor network means the ability of communicating with all of hetwork nodes. Thus, we will define the complete connectivity of the sensor network, $\operatorname{Con}\left(R_{\mathrm{T}}\right)$, as (10):

$$
\operatorname{con}\left(R_{T}\right)=2 \text { Else }
$$

Where $M C P$ is the biggest connected component of the sensor network and $n$ is the number of the sensor nodes. Also $\varepsilon$ shows a very small positive number. Thus, we will define the probability of the complete connectivity, $P_{\mathrm{C}}$, as (11):

$$
\mathrm{P}_{C}=\Sigma_{i=1 \text { to Nd }} \operatorname{Con}_{i}\left(R_{T}\right) / N_{d}
$$

(n (1) $1, N_{d}$ is the number of different configuration of the sensor network.

\section{The Proposed Protocol}

In this section, we propose a topology control solution based on harmony search algorithm and try to decrease the average of transition radius without decreasing the connectivity of the sensor network.

In the proposed algorithm, at first a primary population of transition radii set are selected randomly. Each transition radius set represents one configuration of the sensor network. We 
consider the mentioned population as Harmony Memory (HM). Then we try to improve the harmony memory by creating a new transition radius set. If the new transition radius set is better than the worst transition radius set existing in the harmony memory, the worst one will be replaced with it. The process of providing new transition radius set and replacing the worst transition radius set with the new one continue until meeting termination criteria. Algorithm continues until achieving a certain number of iteration. Finally, the best transition radius set existing in the harmony memory is selected as the response. So, the proposed algorithm is presented briefly in some steps as follows:

Phase 1. The problem and the algorithm parameter initialization:

Step1: Initialize $A_{\min }, A_{\mathrm{T}}$ and $A_{\max }$ sets for each node.

Step2: Produce lower bound and upper bound of the transition radius for each node.

Step3: Initialize the algorithm parameters.

Step4: Initialize the harmony memory with the transition radius ets 1andomly.

Phase2. Repeat the main loop of algorithm until meeting termination criteria:

Step5: Improvise a new transition radius set.

Step6: Update the harmony memory.

Step7: Check the stopping criterion.

In next sections, we describe the proposed algorithm in detail.

\subsection{Step1: Initialize $A_{\min }, A_{\mathrm{T}}$ and $A_{\text {max }}$ ets for each node}

At first, according to (12), the transition Tadius of each node is set between $R_{\min }$ and $R_{\max }$.

$$
\forall R_{i} \in R_{T}=\left(R_{1}, R_{2}, R_{3}, \ldots, R_{h}\right): R_{i} \leftarrow R_{t}
$$

As mentioned before, $R t$ is determined proportional to the network density [20].

\subsection{Step2: Produce $\mathrm{Lower}$ Bound and Upper Bound of the Transition Radius for each Node}

The process of calculating lower bound and upper bound of the transition radius for each node $n_{i} \in S=\left\{n_{1}, n_{2}, \ldots, n_{n}\right\}$ is as lollows:

At first, according toly as mentioned before, $A_{\min }, A_{\mathrm{T}}$ and $A_{\max }$ sets for all nodes is created. Then, $A_{\min }, A_{\mathrm{T}}$ and $A_{\max }$ sets are updated for node $n_{i}$. For this purpose, according to (13), whenever one node of $A_{\mathrm{T}}\left(n_{i}\right)$ and $A_{\max }\left(n_{i}\right)$ sets is accessible through nodes that are in $A_{\min }\left(n_{i}\right)$ set, that will be removed from these sets and adds to $A_{\min }\left(n_{i}\right)$ set (see Figure 1(a)). Also, whenever one node of $A_{\max }\left(n_{i}\right)$ sets is accessible through nodes that are in $A_{\mathrm{T}}\left(n_{i}\right)$ set, that will be removed from $A_{\max }\left(n_{i}\right)$ set and adds to $A_{\mathrm{T}}\left(n_{i}\right)$ set (see Figure $1(b)$ ).
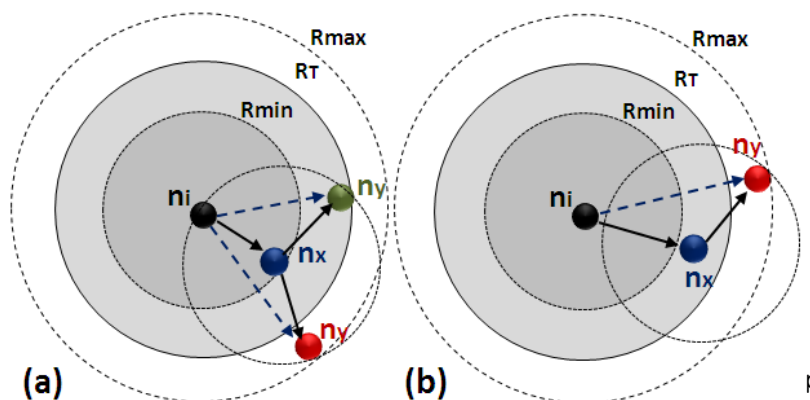

(b) 
Figure 1. Updating the $A_{\min }, A_{T}$ and $A_{\max }$ Sets

$$
\begin{aligned}
n_{i} \in S=\left\{n_{1}, n_{2}, n_{3}, \ldots,\right. & \left.n_{n}\right\}: \\
& \forall n_{x} \in A_{\min }\left(n_{i}\right) \exists n_{y} \in A_{\min }\left(n_{x}\right) A N D \\
& \left(n_{y} \in A_{T}\left(n_{i}\right) \text { OR } n_{y} \in A_{\max }\left(n_{i}\right)\right) \Rightarrow \\
& \left\{\begin{array}{l}
A_{\min }\left(n_{i}\right) \leftarrow A_{\min }\left(n_{i}\right)+n_{y} \\
A_{T}\left(n_{i}\right) \leftarrow A_{T}\left(n_{i}\right)-n_{y} \text { OR } A_{\max }\left(n_{i}\right) \leftarrow A_{\max }\left(n_{i}\right)-n_{y}
\end{array}\right. \\
& \forall n_{x} \in A_{T}\left(n_{i}\right) \exists n_{y} \in A_{\min }\left(n_{x}\right) \text { AND } n_{y} \in A_{\max }\left(n_{i}\right) \Rightarrow \\
& \left\{\begin{array}{l}
A_{T}\left(n_{i}\right) \leftarrow A_{T}\left(n_{i}\right)+n_{y} \\
A_{\max }\left(n_{i}\right) \leftarrow A_{\max }\left(n_{i}\right)-n_{y}
\end{array}\right.
\end{aligned}
$$

In (13), $A_{X}\left(n_{i}\right)$ shows the $A_{X}$ set of node $n_{i}$. Then, regarding $A_{\mathrm{T}}\left(n_{i}\right)$ and $A_{\text {max }}\left(n_{i}\right)$ conditions, we determine the transition radius range for node $n_{i}$. The method of determining the transition radius range is calculated according to the four conditions:

$$
\begin{gathered}
n_{i} \in S=\left\{n_{1}, n_{2}, n_{3}, \ldots, n_{n}\right\}: \\
\text { If } A_{T}\left(n_{i}\right)=\varphi \text { And } A_{\text {max }}(n)=\varphi \text { Then } \\
\text { Else If } A_{T}\left(n_{i}\right) \neq \varphi \text { And } A_{\text {max }}\left(n_{i}\right)=\varphi \text { Then } \\
R_{\text {range } i}=\left[R_{\text {min }} R_{i}\right]=\left[R_{\text {min }}, R_{t}\right] \\
\text { Else If } A_{T}\left(n_{i} \neq \varphi \text { And } A_{\text {max }}\left(n_{i}\right)=\varphi\right. \text { Then } \\
\text { Else If } A_{T}\left(n_{i}\right) \neq \varphi \text { And } A_{\text {max }}\left(n_{i}\right) \neq \varphi \text { Then }
\end{gathered}
$$

Thus, we perform a transition radius ranges set, $R_{\text {range }}$, as:

$$
R_{\text {rang }}=\left\{R_{\text {range } 1}, R_{\text {rang }} \hat{2}, \ldots, R_{\text {range }} \hat{n}\right\}
$$

Now, the transition radius of each node can be only within its determined range:

$$
\forall n_{i} \in S=\left\{n_{l}, n_{2}, ., n_{n}\right\}: R_{i} \in R_{\text {range } i} \hat{i}=\left[R_{\text {low } i} \hat{,} R_{\text {up } i}\right]
$$

\subsection{Step3. Inithalize the Algorithm Parameters}

In this tep, the optimization problem is specified as follows:

$$
\text { Maximize } f\left(R_{T}\right)=\operatorname{Con}\left(R_{T}\right) \times\left(1 /\left(E_{\text {total }}\left(R_{T}\right)+\gamma\right)\right)
$$

Where $R_{\mathrm{T}}$ is the transition radius set; $\gamma$ shows very small positive number that should be selected properly such a way that $f\left(R_{\mathrm{T}}\right)$ function value doesn't exceed the threshold value. The amount of function Con $\left(R_{T}^{i}\right)$ is calculated according to (10).

As mentioned before, $E_{\text {total }}\left(R_{\mathrm{T}}\right)$ is the transition energy consumption of the sensor network based on (8), so:

$$
\begin{aligned}
\text { If Con }\left(R_{T}\right)=1 \Rightarrow f\left(R_{T}\right)=\left(1 /\left(E_{\text {total }}\left(R_{T}\right)+\gamma\right)\right) \Rightarrow & (18) \\
\text { Maximum } f\left(R_{T}\right)=f\left(R_{\text {MIN }}\right), R_{\text {MIN }} & =\left(R_{\text {min }}, R_{\text {min }}, \ldots R_{\text {min }}\right) .
\end{aligned}
$$


Therefore, according to (18), $f\left(R_{\mathrm{T}}\right)$ function value is in inverse ratio to the energy consumption.

Also, the HS algorithm parameters are also initialized in this step [14]:

- HMS: The harmony memory size (or the number of the transition radius sets in the harmony memory.

- HMCR: The harmony memory considering rate.

- PAR: pitch adjusting rate.

- NI: The number of improvisations, or stopping criterion.

\subsection{Step4.Initialize the Harmony Memory}

The Harmony Memory $(H M)$ is a memory location where all transition radius sets are stored. In this step, we consider a Harmony Memory consists of one HMS group of the transition radius sets according to Fig. 2. Each the transition radius set, $R_{\mathrm{T}}^{i}=\left(R_{1}{ }^{i}, R_{2}{ }^{i}, \ldots, R_{n}{ }^{i}\right)$, represents one configuration of sensor network such a way that the transition radius of each $j$ th node equals to $R_{j}$. The fitnes yalue of this configuration is shown by $f\left(R_{\mathrm{T}}^{i}\right)$.

$$
\mathrm{HM}=\left[\begin{array}{ccccc|c}
R_{1}^{1} & R_{2}^{1} & \cdots & R_{n-1}^{1} & R_{n}^{1} & f\left(R^{1}\right) \\
R_{1}^{2} & R_{2}^{2} & \cdots & R_{n-1} & R_{n}^{2} & f\left(R^{2}\right) \\
\vdots & \vdots & \vdots & \vdots & \vdots & \vdots \\
R_{1}^{H M S-1} & R_{2}^{H M S-} & R_{n-1}^{H M S-1} & R_{n M S-1} & f\left(R^{H M S-1}\right) \\
R_{1}^{H M S} & R^{H M S} & \cdots & R_{n-1}^{H N} & R_{n}^{H M S} & f\left(R^{H M S}\right)
\end{array}\right]
$$

Figure 2. The Harmony Memoly (HM) is a Memory Location where all Transition

$$
\text { Radius Sets are Stored }
$$

So, one $n \times H M S$ matrix of transition radii will be obtained. According to (19), these transition radii are initralized randomly:

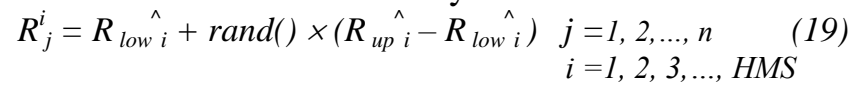

Where $\operatorname{rand}()$ is a randon number between 0 and 1 . HMS is harmony memory size (i.e., the number of the transition radius sets in the harmony memory) and $n$ is the number of nodes.

The fitnes $\$$ value of each transition radius set $R_{\mathrm{T}}^{i}$ is represented by $f\left(R_{\mathrm{T}}^{i}\right)$ and it is calculated by (20):

$$
\begin{array}{r}
\left(R^{i}\right)=\operatorname{Con}\left(R_{T}^{i}\right) \times\left(1 /\left(E_{\text {total }}\left(R_{T}^{i}\right)+\gamma\right)\right) \\
R_{T}^{i}=\left(R_{1}^{i}, R_{2}^{i}, R_{3}^{i}, \ldots, R_{N}{ }^{i}\right), i=1,2,3, \ldots, H M S
\end{array}
$$

As onentioned before, $E_{\text {total }}\left(R_{\mathrm{T}}^{i}\right)$ is the transition energy consumption based on (8) and the amount of function $C\left(R_{\mathrm{T}}^{i}\right)$ is calculated according to (10).

\subsection{Step5. Improvise a Transition Radius Set}

Generating a new transition radius set is called 'improvisation'. A new transition radius set, $R_{\mathrm{T}}^{\prime}=\left(R_{1}^{\prime}, R_{2}^{\prime}, \ldots, R_{\mathrm{N}}^{\prime}\right)$, is generated based on three rules [14]:

- Memory consideration

- Random selection 
- Pitch adjustment

In this step, memory consideration, pitch adjustment or random selection is applied to each radius of the new transition radius set $R_{\mathrm{T}}^{\prime}=\left(R_{1}^{\prime}, R_{2}^{\prime}, \ldots, R_{n}^{\prime}\right)$ in turn [14]. Each variable of the new transition radius set, $R_{i}^{\prime} \in R^{\prime}$, is calculated as follows:

In the memory consideration, the value of the transition radius $R_{i}^{\prime}$ is chosen from any of the transition radius values in the specified harmony memory range $\left({R^{\prime}}_{i}{ }_{i}, R_{i}^{\prime 2}, \ldots, R_{i}^{\prime \mathrm{HMS}}\right)$. The $H M C R$ is the rate of choosing one value from the historical transition radius values stored in the harmony memory.

Also, every transition radius $R_{i}^{\prime} \in R^{\prime}{ }_{\mathrm{T}}=\left\{R_{1}^{\prime}, R^{\prime}{ }_{2}, \ldots, R_{n}^{\prime}\right\}$ obtained by the memory consideration is examined to determine whether it should be pitch-adjusted [14]:

$$
\begin{aligned}
& \text { Pitch adjusting decision for } R_{i}^{\prime} \in R_{T}^{\prime}=\left(R_{l}^{\prime}, R_{2}^{\prime}, \ldots, R_{n}^{\prime}\right) \text { : } \\
& \qquad R_{i}^{\prime}=\left\{\begin{array}{cc}
\text { Yes } & \text { with probability } P A R \\
\text { No } & \text { with probability }(1-P A R)
\end{array}\right.
\end{aligned}
$$

$P A R$ parameter is the rate of the pitch adjustment. The value of PAR defines the rate of doing pitch adjustment, and the value of $(1-P A R)$ defines the rate of doing nothing. If the pitch adjustment is applied, $R_{i}^{\prime}$ is changed as follow:

$$
R_{i}^{\prime} \leftarrow R_{i}^{\prime} \pm \operatorname{rand}() \times\left(\left(R_{\text {up } i}-R_{\text {low } i}\right) \times \mu\right)
$$

Where $\mu$ is a constant factor between 0 and 1 (eg. $\mu=0.5$ )

In the random selection, the value of the transition radius $R_{i}^{\prime}$ is chosen from the possible range of values randomly (i.e., one value between $\left.\left[R_{\text {low } i}, R_{\text {up }} \hat{i}\right]\right) .(1-H M C R)$ is the rate of the random selection. Thus:

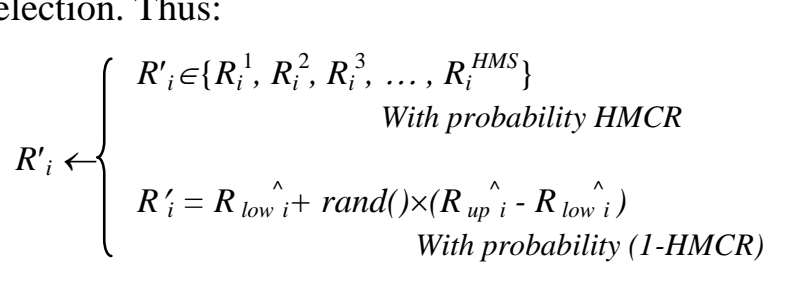

Where $\operatorname{rand}()$ is a randôm aumber between 0 and 1 . Also, as already stated, $R_{\text {low } i}$ and $R_{\text {up } i}$ are the lower and upper bounds for transition radius of node $n_{i}$.

\subsection{Step6. Update the Harmony Memory}

If the new transition radius set, $R_{\mathrm{T}}^{\prime}=\left(R_{1}^{\prime}, R_{2}^{\prime}, \ldots, R_{n}^{\prime}\right)$, is better than the worst transition radius set existing in the harmony memory, the worst one will be replaced with it.

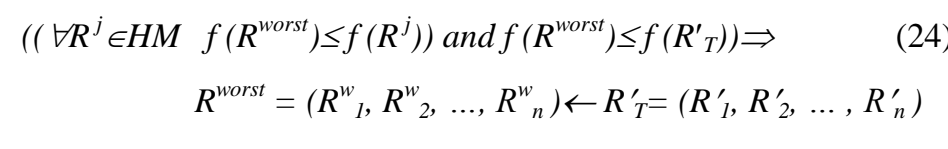

\subsection{Step7. Check stopping criterion}

The main loop of algorithm continues until achieving a certain number of iteration. According to (25), after terminating the main loop of algorithm, the transition radius set with most objective function is selected among transition radius sets existing in the harmony memory.

$$
R^{\text {Best }}=\left(R_{1}^{\text {Best }}, R_{2}^{\text {Best }}, \ldots, R_{N}^{\text {Best }}\right) \text { is answer if: }
$$




$$
\exists R^{\text {Best }} \in H M: \forall R^{j} \in H M \Rightarrow f\left(R^{\text {Best }}\right) \geq f\left(R^{j}\right)
$$

Thus, according to (26):

$$
\begin{aligned}
& R^{\text {Best }}=\left(R_{1}{ }^{\text {Best }}, R_{2}{ }^{\text {Best }}, \ldots, R_{\mathrm{N}}{ }^{\text {Best }}\right) \text { is answer if: } \\
& \forall n_{i} \in S=\left\{\hat{n_{1}}, \hat{n_{2}}, \ldots, \hat{n_{n}}\right\}: R_{i} \leftarrow R_{i}{ }^{\text {Best }}
\end{aligned}
$$

\section{Simulation Results}

In this section, our proposed protocol is simulated and compared to $R A A-2 L, R A A-3 L$ [21], and $H O M$ [20] using NS2 simulator.

\subsection{Simulation Environment}

We considered $500 \times 500 \mathrm{~m}^{2}$ area for these simulations. We deploy the sensor nodes randomly in the target area. The number of sensor nodes, $n$, in different configurations is considered from 50 to 250 sensor nodes. Each node has a transition range between $R_{\min }$ and $R_{\max } . R_{\min }$ and $R_{\max }$ transition radius are considered $0.8 \times R t$ and $1.25 \times R t$ pespectively. Table I represents the $R t, R_{\min }$ and $R_{\max }$ transition values for different configurations of the sensor network. The threshold value for the number of performing the main loop of the algorithm considered $300(N I=300)$. The parameters values for simulation are shown in Table II. The results mentioned in next sections show the average of performing protocols for one hundred random deployments.

\section{Table I. The Minimum and Maximum Transition Radius Values for Simulation}

\begin{tabular}{c|c|c|c|c|c|c|c|c|c}
\hline Number of nodes & 50 & 5 & 100 & 125 & 150 & 175 & 200 & 225 & 250 \\
\hline$R \min$ & 109 & 87 & 77 & 69 & 64 & 59 & 57 & 56 & 55 \\
\hline$R t$ & 87 & 0 & 62 & 55 & 51 & 47 & 46 & 45 & 44 \\
\hline Rmax & 136 & 109 & 96 & 86 & 80 & 74 & 71 & 70 & 69 \\
\hline \\
Table \\
\hline
\end{tabular}

Three metrics are used foryaluations. These metrics are: the average of transition radius, the average number of neighbors, and the probability of the complete connectivity.

\subsection{Comparing with other Protocols}

In the first experiment, we measured average of the transition radius of the network for EETCP, RAA $2 L, R A A-3 L$ and HOM protocols. The purpose of this experiment is to evaluate the ability of the proposed protocol to decrease average of the transition radius. Note that, in $M I N-R A N G E$, all the nodes have minimum transition radius $\left(R_{m i n}\right)$. Also, in $M A X-R A N G E$, all the nodes have maximum transition radius $\left(R_{\max }\right)$. The results of this simulation are depicted in Figure 3(a) and Table III. As can be seen, EETCP has less average of transition radius and $H O M$ has maximum average of transition radius. Note that, against other protocols, the proposed protocol doesn't use a predetermined transition radius.

In second experiment, the average numbers of neighbors for EETCP, $R A A-2 L, R A A-3 L$ and $H O M$ protocols are measured. The purpose of this experiment is to evaluate the ability of the proposed protocol to decrease the number of neighbors. The results of this experiment are depicted in Figure 3(b) and Table IV. As can be seen in Figure 3(b), the average number of neighbors in the EETCP is less than those of other protocols. Note that a lower number of 
neighbors results in lower interference between the nodes. Decreasing of the neighbors directly results in decreasing the transition radius. But note that decreasing the neighbors and also the transition radius is useful if the network connectivity is remained (is not removed). This problem will be evaluated accurately in next experiment.

In the last experiment, the network connectivity in EETCP is measured and compared to $R A A-2 L, R A A-3 L, H O M, M I N-R A N G E$ and MAX-RANGE protocols. As mentioned before, in these experiments, we supposed $N_{d}$ equal to 100 . The probability of the complete connectivity is depicted in Figure 3(c) and Table $\mathrm{V}$ for EETCP, RAA-2L, RAA-3L and HOM protocols. As can be seen in Figure 3(c), the probability of complete connectivity of the network for EETCP, RAA-2L, RAA-3L and MAX-RANGE are almost equal. So, the network connectivity in our protocol is acceptable.

Table III. Average of Transition Radius

\begin{tabular}{c|c|c|c|c|c|c|c|c|c}
\hline Number of nodes & 50 & 75 & 100 & 125 & 150 & 175 & 200 & 225 & 250 \\
\hline$E E T C P$ & 96.2 & 77.6 & 63.3 & 57.1 & 53.7 & 52.2 & 46.1 & 46.3 & 46.1 \\
\hline$R A A-3 L$ & 107.3 & 92.1 & 75.1 & 71.5 & 61.1 & 571 & 52. & 50.1 & 47.4 \\
\hline$R A A-2 L$ & 118.1 & 96.1 & 84.3 & 77.4 & 71.5 & 66. & 60.9 & 57.0 & 52.7 \\
\hline$H O M$ & 109 & 87 & 77 & 69 & 64 & 59 & 57 & 56 & 55 \\
\hline
\end{tabular}

Table IV. Average Number of Neighbors

\begin{tabular}{c|c|c|c|c|c|c|c|c|c}
\hline Number of nodes & 50 & 75 & 100 & 125 & 150 & 175 & 200 & 225 & 250 \\
\hline$E E T C P$ & 6.22 & 5.5 & 5.81 & 5.82 & 5.87 & 6.0 & 6.02 & 6.52 & 7.23 \\
\hline$R A A-3 L$ & 6.92 & 6.72 & 6.82 & 7.0 & 7.64 & 7.63 & 7.64 & 7.88 & 8.39 \\
\hline$R A A-2 L$ & 7.46 & 7.38 & 7.82 & 7.83 & 8.93 & 9.32 & 9.72 & 9.73 & 9.94 \\
\hline$H O M$ & 8.8 & 8.9 & 9.0 & 9.6 & 9.3 & 9.8 & 10.3 & 10.5 & 10.6 \\
\hline$M I N-R A N G E$ & 5.4 & 4.81 & 5.2 & 5.29 & 5.53 & 5.41 & 5.57 & 5.87 & 7.09 \\
\hline$M A X-R A N G E$ & 9.96 & 10.1 & 10.4 & -1 & 12.2 & 12 & 12 & 13.4 & 14.6 \\
\hline
\end{tabular}

Table V. The Probability of the Complete Connectivity

\begin{tabular}{c|c|c|c|c|c|c|c|c|c}
\hline Number of nodếs & 50 & 75 & 100 & 125 & 150 & 175 & 200 & 225 & 250 \\
\hline EETCP & 0.97 & 9.98 & 0.98 & 0.97 & 9.97 & 0.98 & 0.97 & 0.97 & 0.98 \\
\hline RAA-3L & 0.98 & 0.99 & 0.98 & 0.98 & 0.98 & 0.98 & 0.98 & 0.97 & 0.98 \\
\hline RAA-2L & 0.99 & 0.99 & 0.98 & 0.99 & 0.98 & 0.99 & 0.98 & 0.99 & 0.98 \\
\hline$H O M$ & 0.06 & 0.08 & 0.07 & 0.07 & 0.05 & 0.06 & 0.07 & 0.05 & 0.08 \\
\hline MIN-RANGE & 0.02 & 0.02 & 0.03 & 0.02 & 0.01 & 0.01 & 0.02 & 0.00 & 0.00 \\
\hline MAX-RANGE & 0.98 & 0.99 & 1.00 & 1.00 & 1.00 & 1.00 & 1.00 & 1.00 & 1.00 \\
\hline
\end{tabular}
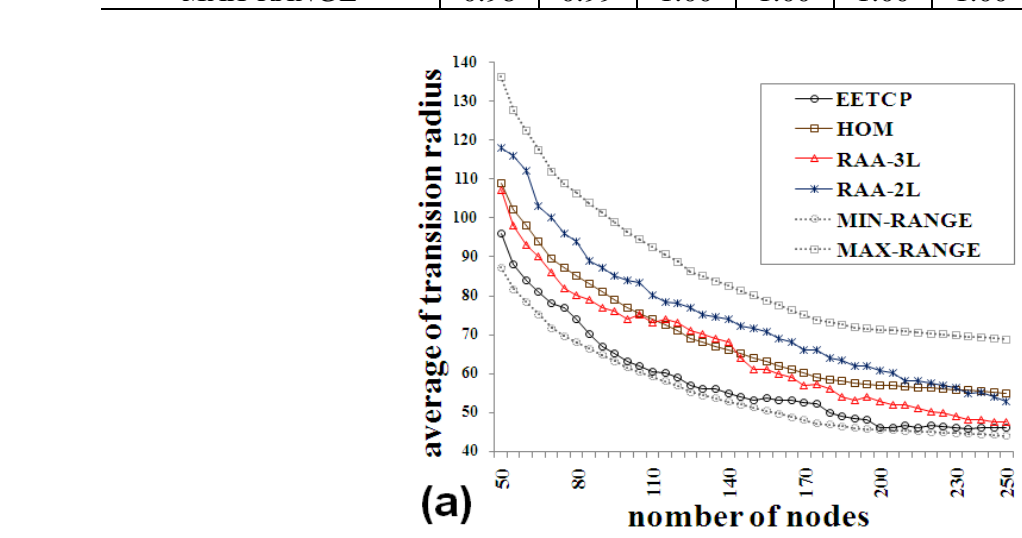

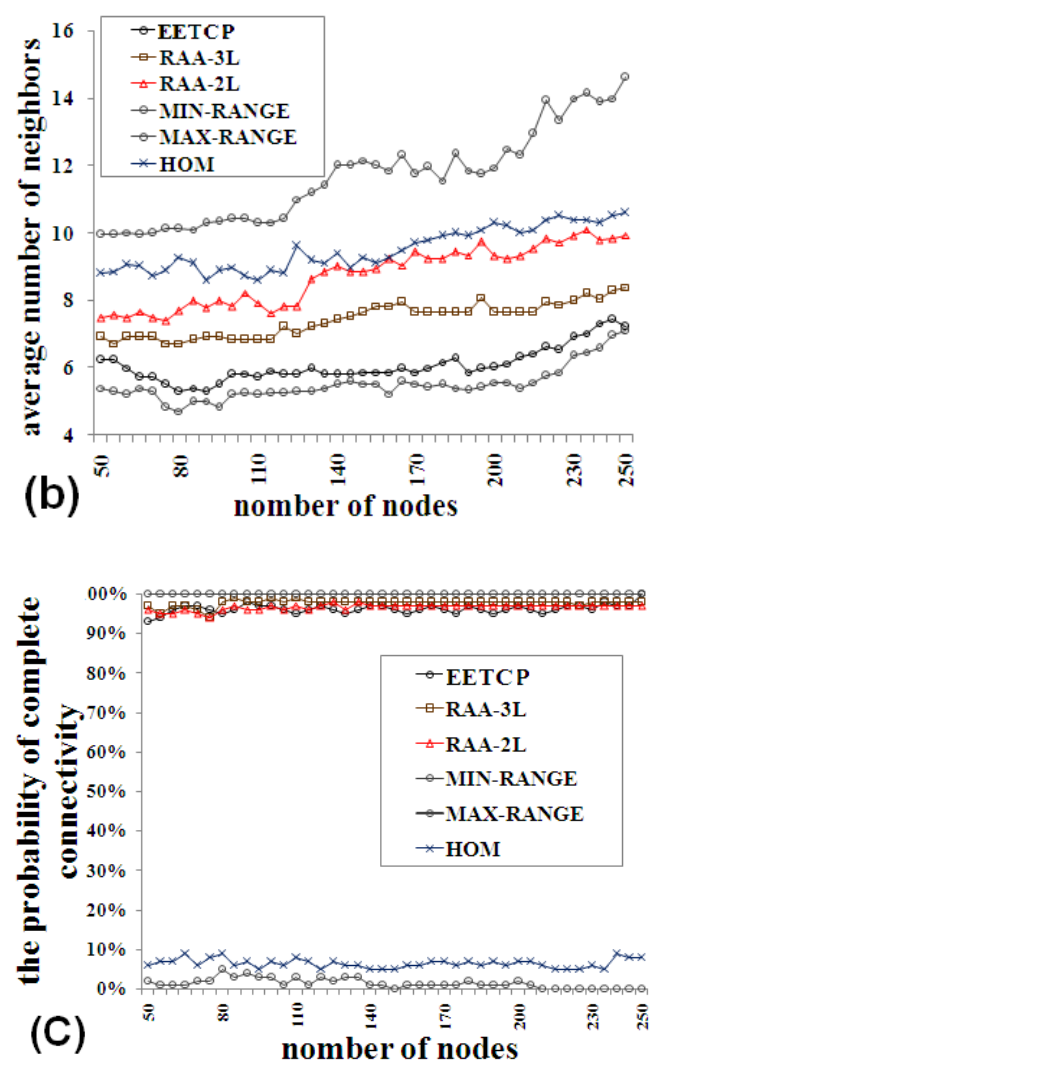

Figure 3. (a) Average of the Transition Radius, (b) Average Number of Neighbors, (c) Probability of the Complete

\subsection{Observations}

As can be seen in Figure 3, while hodes have the minimum transition radius $\left(R_{\text {min }}\right)$, the state of the network connectivity is very undesirable. Also while the nodes have maximum transition radius $\left(R_{\max }\right)$, the nomber of network links are lot such a way that not only the energy consumption is very high but also the collision within transition radius is lot.

These results can show the prominence of our proposed protocol. While maintaining network connectivity (1t could decrease the average of transition radius and the average number of neighbor nodes. Thus it decreases the energy consumption and the interference between sensor nodes. EETCP protocol has some advantages compared to the previous protocols. EETCP protocol dynamically adjusts the transition radius of the nodes (unlike previous frotocols which should select radius values among predefined values). Thus, our protocol has the less average number of neighbors compared to the existing protocols. Also, the energy consumption in our protocol is less than others and the network lifetime will be prolonged. In addition, the network connectivity in our protocol is in the acceptable level.

\section{Conclusion}

In this paper, we produced a cluster-based topology control scheme and proposed a new Energy Efficient Topology Control Protocol named EETCP. In EETCP protocol, proper transition radius determined using HS algorithm. Unlike previous protocols that should select the transition radius values among predefined values, EETCP dynamically adjusts transition 
radius of nodes. Thus, the proposed protocol has some advantage compared to previous protocols. EETCP has less average number of neighbors compared to existing protocols. Also, the energy consumption in EETCP is less than others and the network lifetime will be prolonged. In addition, the network connectivity in EETCP is in the acceptable level. The proposed protocol is simulated and the above advantages are shown in the simulation results.

\section{References}

[1] M. Al Ameen and K. Kwak, "Social Issues in Wireless Sensor Networks with Healthcare Perspective", The International Arab Journal of Information Technology, vol. 8, no. 1, (2011) January.

[2] B. Alatas, "Chaotic harmony search algorithms", Elsevier, Applied Mathematics and Computation 216, (2010), pp. 2687-2699.

[3] S. Ali and S. Madani, "Distributed Grid based Robust Clustering Protocol for Mobile Sensor Networks", The International Arab Journal of Information Technology, vol. 8, no. 4, (2011) October.

[4] D. Blough, M. Leoncini, G. Resta and P. Santi, "The k-neighbors protocol for symmetric topology control in ad hoc networks", Proceedings of the ACM MobiHoc 03, (2003), pp. 141-152.

[5] N. Bulusu, J. Heidemann and D. Estrin, "GPS-less low-cost outdoor localization for very small devices", IEEE Personal Communications, vol. 7, pp. 28-34,2000.

[6] Z. Geem, J. Kim and G. Loganathan, "A new heuristic optimization algorithm: harmony search", Elsevier, Simulation, vol. 76, no. 2, (2001), pp. 60-68.

[7] S. M. Jamei, A. Nikdel and A. M. Bidgoli, "GATC: A new Genetic Algorithm-based Topology Control Protocol for Wireless Sensor Networks", The International Conference on Intelligent Network and Computing (ICINC 2010), Kuala Lumpur, Malaysia during, (2010) November 26-28.

[8] S. Jardosh and P. Ranjan, "A Survey: Topology Control for Wireless Sensor Networks", Proceeding of the International Conference on Signal processing Communications and Networking Madras Institute of Technology (IEEE), Anna University Chennai India, (2008) January 4-6, pp. 422-427.

[9] J. Jia, J. Chen, Y. Wen and G. Chang, "Ap-extensible care-control routing protocol in large scale ad-hoc networks", Proceedings of the 6th International Conference on TTS Telecommunications, Chengdu, China, (2006), pp. 955-958.

[10] J. Jia, J. Chen, G. Chang, Y. Wen and Y Song, "Multiobjective optimization for coverage control in wireless sensor network with adjustablesensing radius", Êlsevier Computers and Mathematics with Applications, vol. 57, (2009), pp. 1767-1775.

[11] N. Li, J. Hou and L. Sha, "Design and analysis of an mst-based topology control algorithm", Proceedings of the IEEE Infocom, vol. 4, (2005) May, pp. 1195-1206.

[12] X. Li, Y. Mao and Y Liang, "A Survey on Topology Control in Wireless Sensor Networks", 10th International Conference on Control, Automation, Robotics and Vision, ICARCV, Hanoi, Vietnam, (2008) December 17-20

[13] M. Lu, J. Wu, M. Cardei and-M. Li, "Energy-efficient connected coverage of discrete targets in wireless sensor networks", Proceedings of the International Conference on Computer Networks and Mobile Computing, ICCNMC, Zhangjiajie, China, (2005), pp. 43-52.

[14] M. Mahdavi, M. Fesanghary and E. Damangir, "An improved harmony search algorithm for solving optimization problems" Elsevier, Applied Mathematics and Computation, vol. 188, (2007), pp. 1567-1579.

[15] S. Narayanswamy, V. Kawadia, R. S. Sreenivas and P. Kumar, "Power Control in Ad-Hoc networks: Theory, Architecture, Algorithm and Implementation of the COMPOW Protocol", European Wireless ConferenceNext Gêneration Wireless Networks: Technologies, Protocols, Services and Applications. Florence, Italy, (2002) February 25-28.

[16] M. G. H. Omran and M. Mahdavi, "Global-best harmony search", Elsevier, Applied Mathematics and Computation, vol. 198, (2008), pp. 643-656.

[11] Cia, F. Xia, L. Feng, G. Wu and B. Jin, "Queueing theory-based path delay analysis of wireless sensor networks", Advances in Electrical and Computer Engineering (AECE), vol. 11, no. 2, (2011).

[18] V. Rodoplu and T. H. Meng, "Minimum energy mobile wireless networks", Proceedings of the IEEE Journal on Selected Areas in Communications, vol. 17, (1999), pp. 1333-1344.

[19] P. Santi, "Topology Control in Wireless Ad Hoc and Sensor Networks", ACM Computer Survey, vol. 37, no. 2, (2005), pp. 164-194.

[20] D. Stauffer and A. Aharony, "Introduction to Percolation Theory", London: Taylor \& Francis, (1994).

[21] A. Venuturumilli and A. Minai, "Obtaining Robust Wireless Sensor Networks Throuh Self-Organization of Heterogeneous Connectivity", Proceedings of the 2006 International Conference on Complex Systems (ICCS'06), Boston, MA, (2006) June. 
[22] R. Wattenhofer, L. Li, P. Bahl and Y. Wang, "Distributed topology control for power efficient operation in multihop wireless ad hoc networks", Proceedings of the IEEE Infocom, vol. 3, (2001), pp. 1388-1397.

[23] R. Wattenhofer and A. Zollinger, "XTC: a practical topology control algorithm for ad-hoc networks", Proceedings of the 18th International Parallel and Distributed Processing Symposium, (2004) April 26-30, pp. 2-16.

[24] X. Zhang, X. Ding, S. Lu and G. Chen, "Principles for Energy-Efficient Topology Control in Wireless Sensor Networks", WiCom '09. 5th International Conference on Wireless Communications, Networking and Mobile Computing, IEEE, (2009) September 24-26.

\section{Authors}

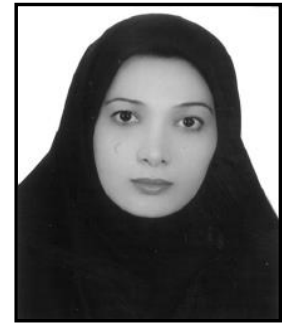

Hagar Noori, received the B.S. degree in Computer Engineering from the Islamic Azad University and the M.S. degrees in Computer Engineering from Arak Science and Research Branch in Iran in 2007 and 2012, respectively. Her research interests include wireless sensor and Ad-hoc networks.

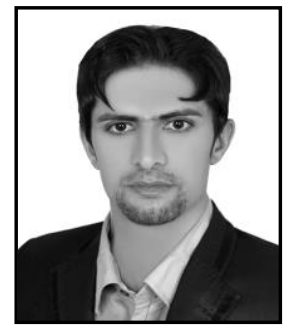

Arash Nikdel, received the B.S. degree in Computer Engineering from the Islamic Azad University and the M.S. degrees in Computer Engineering from Ahyaz Scrence and Research Branch in Iran, in 2007 and 2011, respectively. His research interests include wireless sensor and Ad-hoc networks.
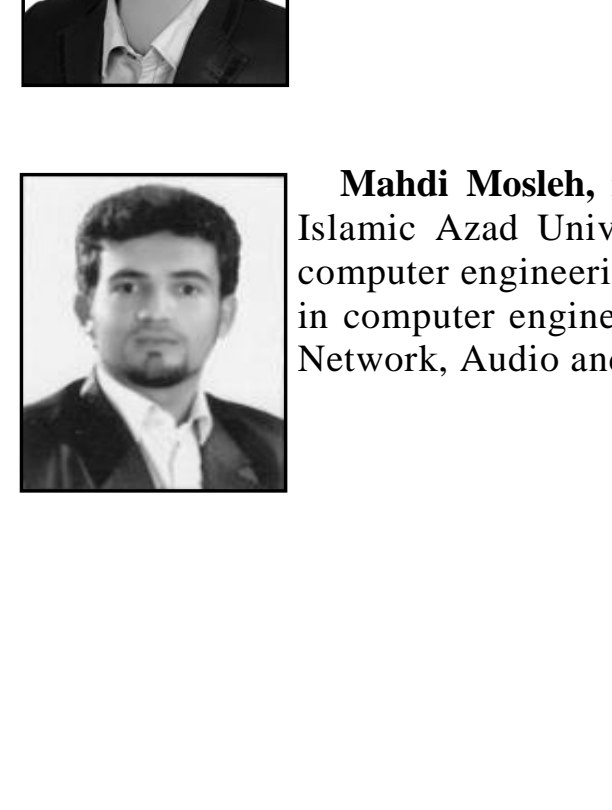

Mahdi Mosleh, received his B.S. in computer engineering from Islâmic Azad University, Dezful Branch, in 2008 and the M.S. in computer engineening from Islamic Azad University, Dezful, in 2011 in computer engineering. His research interests are Wireless Sensor Networl Audio and Image watermarking. 